\title{
Charge-Transfer Dynamics in Nanorod Photocatalysts with Bimetallic Metal Tips
}

\author{
Maria Wächtler $^{\mathrm{a}^{*}}$, Philip Kalisman ${ }^{\mathrm{b \#}}$, Lilac Amirav ${ }^{\mathrm{b}}$ \\ ${ }^{a}$ Leibniz Institute of Photonic Technology Jena, Albert-Einstein-Straße 9, 07745 Jena, Germany \\ ${ }^{b}$ Schulich Faculty of Chemistry, Technion-Israel Institute of Technology, Haifa 32000, Israel
}

\section{Supplemental Information}




\section{Experimental Details}

\section{1. fs time-resolved transient absorption}

The fs time-resolved measurements were performed on as system consisting of a Ti:sapphire amplifier (Legend-Elite, Coherent inc.), producing pulses centred at $795 \mathrm{~nm}$ with a repetition rate of $1 \mathrm{kHz}$. The pump pulses centered at $390 \mathrm{~nm}$ and a duration of $100 \mathrm{fs}$ are generated by second harmonic generation from the fundamental. The white light continuum between 450 and $700 \mathrm{~nm}$ to probe the sample is generated by focussing a part of the fundamental of the amplifier output into a sapphire plate. The pump pulses are delayed with respect to the probe pulses by means of an optical delay stage (maximum delay: $2 \mathrm{~ns}$ ) and focused into the sample by a lens ( $\mathrm{f}=1 \mathrm{~m}$ ). The repetition rate of the pump pulses is reduced to $500 \mathrm{~Hz}$ by a mechanical chopper and the polarization of the pump with respect to the probe pulses is set to the magic angle $\left(54.7^{\circ}\right)$ using a Berek compensator and a polarizer. The white light continuum is split into probe and reference. The probe pulse is focused onto the sample by a concave mirror ( $\mathrm{f}=500 \mathrm{~mm}$ ) and spatially overlapped with the pump pulse. Probe and reference are collected by a detection system (Pascher Instruments, AB) consisting of a spectrograph (Acton, Princeton Instruments) equipped with a double-stripe diode array detector. The diode array is read out with the laser repetition rate and the signal $(\Delta \mathrm{A})$ is calculated from two consecutive probe pulses, corresponding to pump-on and pump-off conditions.

The setup for the TA measurements with a large delay range up to $12 \mathrm{~ns}$ has been described in detail previously. ${ }^{1}$ In short the output of an amplified Ti:Sapphire oscillator (Libra, Coherent Inc.), producing pulses with a repetition rate of $1 \mathrm{kHz}$ centered at $800 \mathrm{~nm}$ (80 fs, $950 \mathrm{~mJ}$ ), was used. The pump pulses at $400 \mathrm{~nm}$ with a duration if $200 \mathrm{fs}$ were produced by second harmonic generation. A chopper was inserted into the pump path to block every second pump pulse. The 
probe light was generated by focusing a part of the fundamental into a sapphire plate to generate a supercontinuum. The probe pulses were delayed in time with respect to the arrival of the pump pulses at the sample position by means of a delay line and split by a beamsplitter to obtain probe and reference pulses. The probe pulses were focused into the sample by means of a spherical mirror. Probe-pulses were spatially and temporally overlapped at the sample position with the pump pulses, which were focused into the sample by means of a $1000 \mathrm{~mm}$ focal-length quartz lens. The polarization of the pump pulses was rotated to yield magic-angle. The probe pulse is re-collimated by a second spherical mirror and in concert with the reference beam sent to the detection system, which allows for simultaneous spectrally resolved detection of probe and reference intensities on a double-stripe diode array. The diode array was read-out with the repetition rate of the laser and the differential absorption signal was calculated from the probe and reference intensities for a pair of measurements corresponding to pump-on and pump-off conditions.

\section{2. ns time-resolved transient absorption}

The setup used for the ns time-resolved measurements has been described previously. ${ }^{2}$ The pump pulses for the ns at $410 \mathrm{~nm}$ were delivered by an OPO (OPO-PLUS, Continuum) pumped by a ND:YAG (Surelite S10 II, Continuum) laser at $10 \mathrm{~Hz}$ resulting in pulses with a duration of $5 \mathrm{~ns}$. Probe light is delivered by a pulsed $75 \mathrm{~W}$ Xe arc lamp. The sample is probed in $90^{\circ}$ geometry. Spherical concave mirrors were used to focus the probe light at the sample position and to refocus the light on the entrance slit of a monochromator (Acton, Princeton Instruments). Probe light is detected by a PMT (Hamamatsu R928) mounted on a fivestage base and the signal was processed by a commercially available detection system (Pascher Instruments AB). 


\section{Transient Spectra}
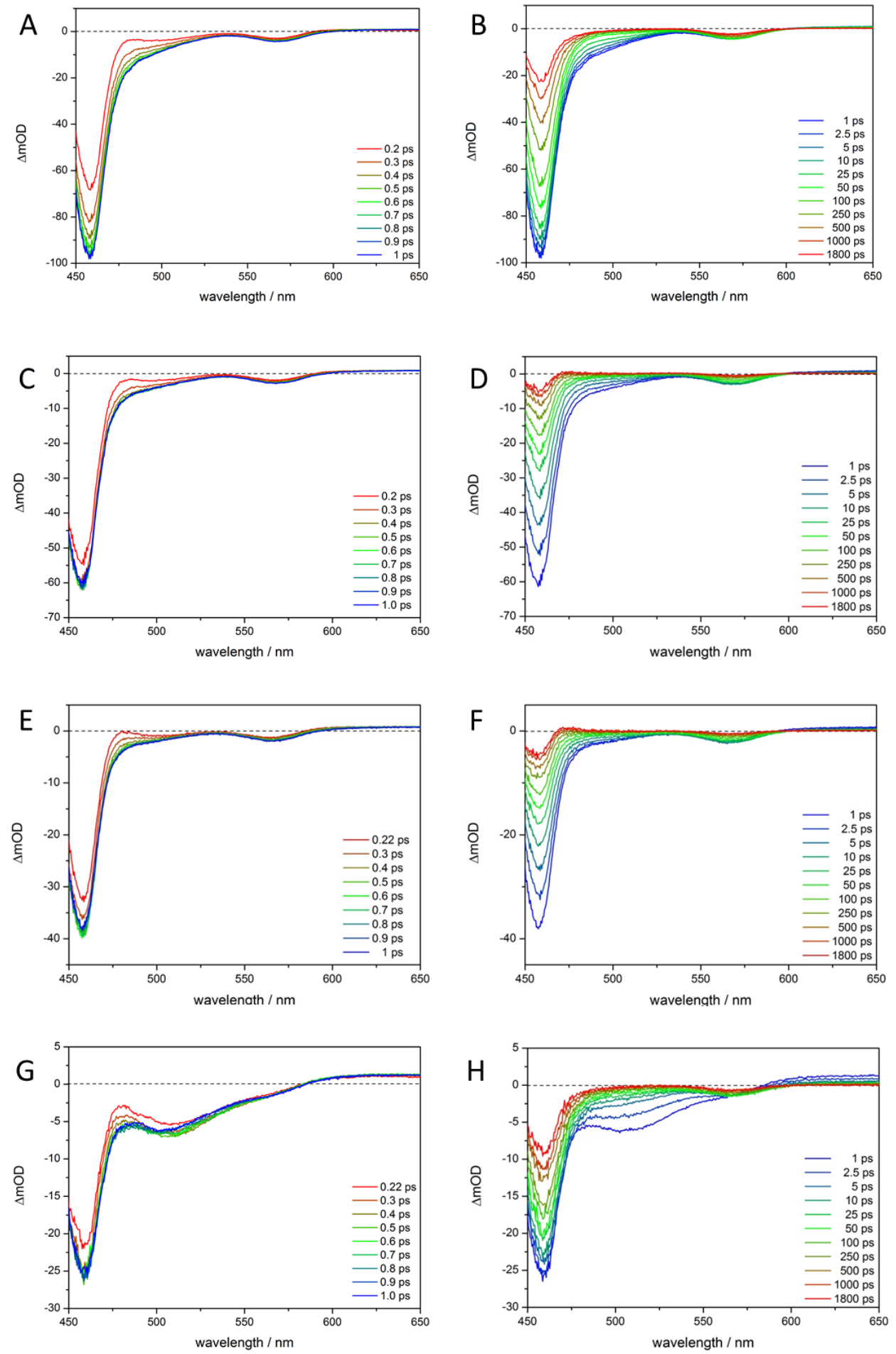

Figure S1: Transient spectra of the bare rod $(A, B)$, the Pt $(C, D), A u P t(E, F)$ and $A u(G, H)$ tipped sample upon excitation at $390 \mathrm{~nm}$. 

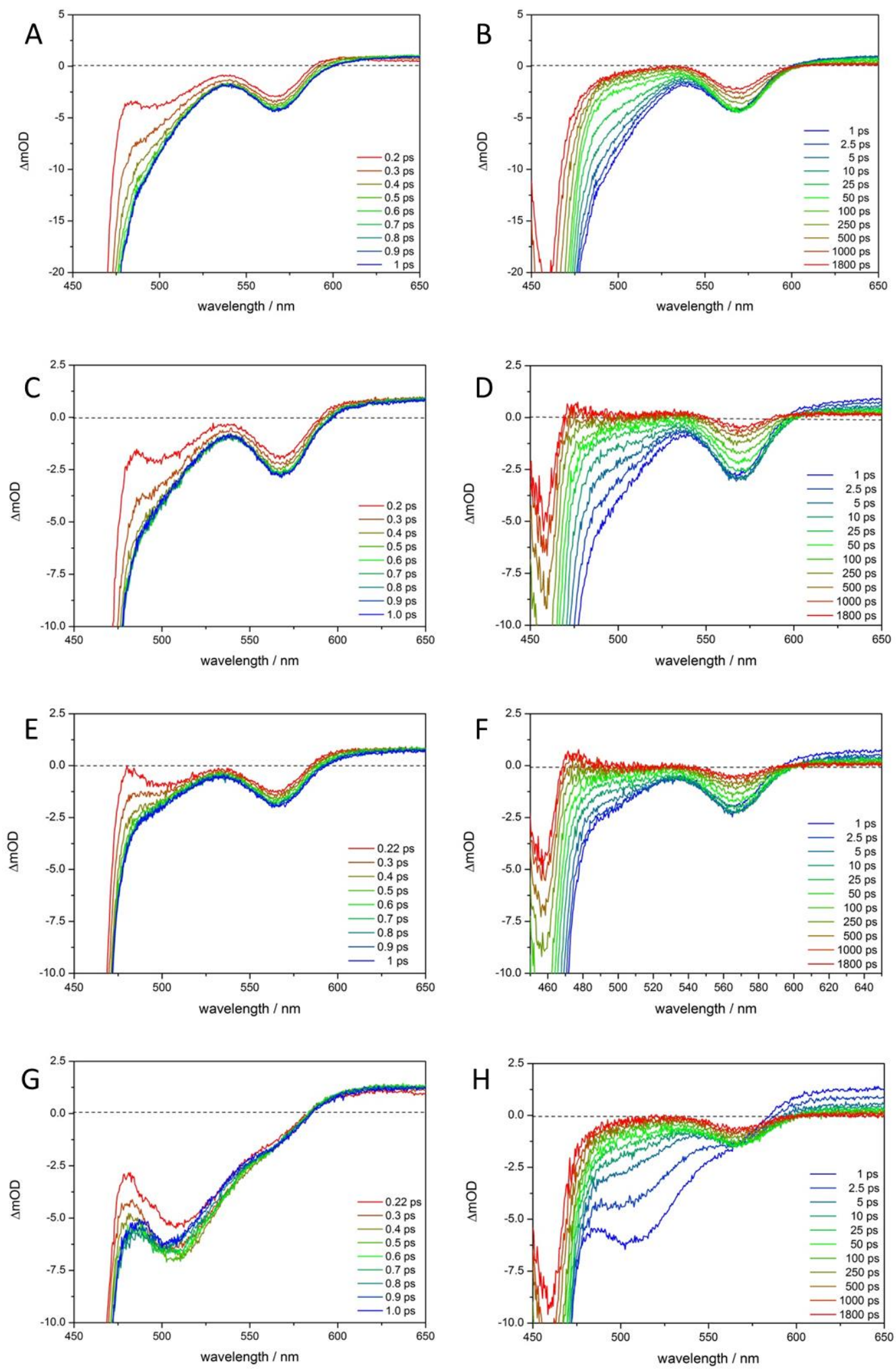

Figure S2: Enlargement of the transient spectra of the bare rod $(A, B)$, the Pt $(C, D), A u P t(E, F)$ and $A u$ $(G, H)$ tipped sample upon excitation at $390 \mathrm{~nm}$. 
The transient spectra of all four samples show the characteristic bleach features of the CdSe and the CdS exciton transitions observable at 570 and $458 \mathrm{~nm}$ in agreement with the static absorption spectra. The bleach feature of the exciton transition in the CdS bulb region is as expected weak due to its low volume and hidden underneath the strong bleach of the exciton transition localized in the CdS rod. At early decay times, a positive signal contribution is superimposed on the strong bleach feature in the red shoulder of the exciton bleach localized in the rod, which decays within the first ps parallel to the increase in the signal intensity of the bleach features, and can be assigned to the presence of hot excitons generated in the nanorod upon excitation into higher excitonic transitions at $400 \mathrm{~nm}^{3-4}$

\section{Fitting results - fs TA data}

\section{Bare rod}

The temporal evolution at chosen probe wavelengths is fitted by applying a sum of exponential functions:

$$
\Delta A(t)=\sum_{i} A_{f i} e^{\frac{-t}{\tau_{f i}}}+\sum_{i} A_{i} e^{\frac{-t}{\tau_{i}}}+\text { const }
$$

$\mathrm{A}_{\mathrm{fi}}, \tau_{\mathrm{fi}}$ amplitude and time constant of signal formation processes

$\mathrm{A}_{\mathrm{i}}, \tau_{\mathrm{i}}$ amplitude and time constant of processes describing the signal decay

Table S1: fit parameters for the bare rods

\begin{tabular}{|l|l|l|l|l|l|}
\hline Bare rod & $\boldsymbol{\tau}_{\mathbf{f} 1} / \mathbf{p s}$ & $\boldsymbol{\tau}_{\mathbf{f} 2} / \mathbf{p s}$ & $\boldsymbol{\tau}_{\mathbf{1}} / \mathbf{p s}$ & $\boldsymbol{\tau}_{\mathbf{2}} / \mathbf{p s}$ & $\boldsymbol{\tau}_{\mathbf{3}} / \mathbf{p s}$ \\
& $\mathbf{A}_{\mathbf{f} 1}$ & $\mathbf{A}_{\mathbf{f} 2}$ & $\mathbf{A}_{1}$ & $\mathbf{A}_{\mathbf{2}}$ & $\mathbf{A}_{\mathbf{3}}$ \\
\hline $458 \mathrm{~nm} \mathrm{CdS}$ & $0.17 \pm 0.01$ & - & $13.5 \pm 0.6$ & $192.1 \pm 4.5$ & $3000^{*}$ \\
& $0.66 \pm 0.04$ & & $-0.13 \pm 0.1$ & $-0.41 \pm 0.01$ & $-0.52 \pm 0.02$ \\
\hline $580 \mathrm{~nm}$ & $0.18 \pm 0.01$ & $21.9 \pm 2.0$ & & $283.9 \pm 37.1$ & $4000^{*}$ \\
$\mathrm{CdSe}$ & $0.92 \pm 0.1$ & $0.22 \pm 0.01$ & & $-0.37 \pm 0.02$ & $-0.75 \pm 0.1$ \\
\hline
\end{tabular}

* fixed value in the fitting procedure, determined by TA measurements with a 10 ns delay line, error $\pm 10 \%$ 


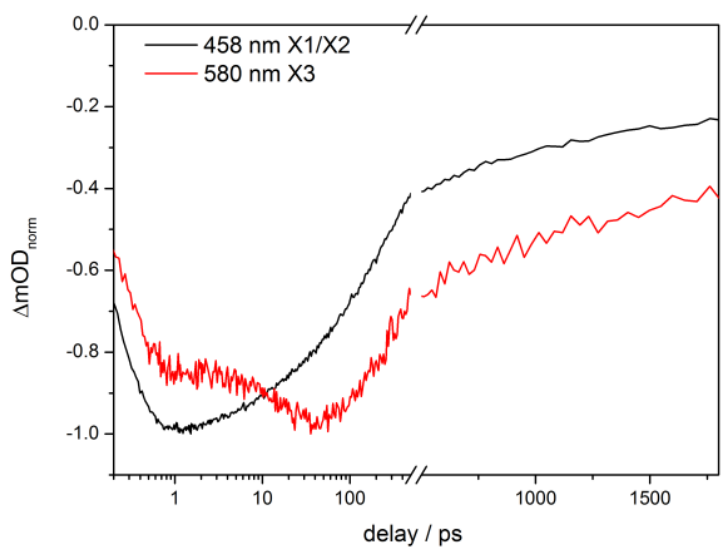

Figure S3: Comparison of the kinetic traces at probewavelenghts $458 \mathrm{~nm}$ and $580 \mathrm{~nm}$ probing CdS rod and shell and CdSe excitonic bleach recovery.

A comparison of the kinetics of the rod localized bleach feature at $458 \mathrm{~nm}$ and the seed localized bleach feature at $580 \mathrm{~nm}$ probe wavelength revels a similar fast formation of the signal, which can be attributed to an electron relaxation process from the initially excited higher excitonic transitions in both the CdS bulk and the CdSe seed, though the latter is only excited to a low extent. The CdSe seed localized excitonic state kinetics shows a second slower formation component (see Table S1), while the $458 \mathrm{~nm}$ rod localized bleach feature is already decaying in this time range. This relates the initial fast decay in the CdS rod bleach and the second growth of the CdSe seed localized exciton signatures to each other and the related process describes the relaxation of an exciton generated in the CdS rod in the CdSe seed. After this process, no interconversion between these two states seems to occur anymore. Similar localization processes have been described before. ${ }^{4}$ 
To model the kinetics of the metal tipped rods, the presence of non-tipped rods and only partial electron transfer was taken in to account by including the rod intrinsic relaxation processes into to the fitting function scaled with a factor $\mathrm{C}$. The formation time, hence the relaxation from hot excitons to the band edge states is assumed to be independent of the absence or presence of the metal tip.

$$
\Delta A(t)=A_{f 1} e^{\frac{-t}{\tau_{f i}}}+\sum_{j} A_{e t j} e^{\frac{-t}{\tau_{e t j}}}+c *\left(\sum_{i} A_{i} e^{\frac{-t}{\tau_{i}}}\right)+\text { const }
$$

$\mathrm{A}_{\mathrm{fi}}, \tau_{\mathrm{fi}}$ amplitude and time constant of signal formation processes

$\mathrm{A}_{\mathrm{i}}, \tau_{\mathrm{i}}$ amplitude and time constant of processes describing the signal decay

$\mathrm{A}_{\mathrm{etj}}, \tau_{\mathrm{etj}}$ amplitude and time constant of electron transfer processes

$\mathrm{C}$ factor for scaling the rod intrinsic relaxation processes

Table S2: fit parameters for electron transfer processes in the Pt tipped sample

\begin{tabular}{|c|c|c|c|c|c|}
\hline $\mathbf{P t}$ & $\begin{array}{l}\tau_{\mathrm{f} 1} / \mathrm{ps} \\
\mathbf{A}_{\mathrm{f} 1}\end{array}$ & $\begin{array}{l}\tau_{\mathrm{f} 2} / \mathbf{p s} \\
\mathbf{A}_{\mathrm{f} 2}\end{array}$ & $\begin{array}{l}\tau_{\mathrm{et} 1} / \mathrm{ps} \\
\mathbf{A}_{\mathrm{et} 1}\end{array}$ & $\begin{array}{l}\tau_{\mathrm{et} 2} / \mathbf{p s} \\
\mathbf{A}_{\mathrm{et} 2}\end{array}$ & C \\
\hline $\begin{array}{l}458 \quad \mathrm{~nm} \\
\mathrm{CdS} \\
\text { (rod/shell) }\end{array}$ & $\begin{array}{l}0.17 \pm 0.01 \\
0.64 \pm 0.02\end{array}$ & - & \begin{tabular}{|l|}
$3.5 \pm 0.1$ \\
$-0.43 \pm 0.01$
\end{tabular} & $\begin{array}{l}34.8 \pm 1.6 \\
-0.23 \pm 0.01\end{array}$ & $0.46 \pm 0.01$ \\
\hline $\begin{array}{ll}580 & \mathrm{~nm} \\
\mathrm{CdSe} & \end{array}$ & $\begin{array}{l}0.18 \pm 0.01 \\
0.75 \pm 0.07\end{array}$ & \begin{tabular}{|l|}
$2.2 \pm 0.3$ \\
$0.28 \pm 0.01$
\end{tabular} & - & $\begin{array}{l}48.3 \pm 2.7 \\
-0.61 \pm 0.01\end{array}$ & $0.69 \pm 0.03$ \\
\hline
\end{tabular}

Table S3: fit parameters for electron transfer processes in the AuPt tipped sample

\begin{tabular}{|c|c|c|c|c|c|}
\hline AuPt & $\begin{array}{l}\tau_{\mathrm{f} 1} / \mathrm{ps} \\
\mathbf{A}_{\mathrm{f} 1}\end{array}$ & $\begin{array}{l}\tau_{\mathrm{f} 2} / \mathrm{ps} \\
\mathbf{A}_{\mathrm{f} 2}\end{array}$ & $\begin{array}{l}\tau_{\text {et1 }} / \mathbf{p s} \\
\mathbf{A}_{\text {et1 }}\end{array}$ & $\begin{array}{l}\tau_{\mathrm{et} 2} / \mathbf{p s} \\
\mathbf{A}_{\mathrm{et} 2}\end{array}$ & C \\
\hline $\begin{array}{l}458 \quad \mathrm{~nm} \\
\mathrm{CdS} \\
\text { (rod/shell) }\end{array}$ & $\begin{array}{l}0.17 \pm 0.01 \\
0.56 \pm 0.03\end{array}$ & - & \begin{tabular}{|l|}
$2.6 \pm 0.1$ \\
$-0.44 \pm 0.01$
\end{tabular} & $\begin{array}{l}31.4 \pm 1.8 \\
-0.23 \pm 0.01\end{array}$ & $0.40 \pm 0.01$ \\
\hline $\begin{array}{ll}580 & \mathrm{~nm} \\
\mathrm{CdSe} & \end{array}$ & $\begin{array}{l}0.18 \pm 0.01 \\
0.34 \pm 0.11\end{array}$ & \begin{tabular}{|l|}
$1.7 \pm 0.2$ \\
$0.41 \pm 0.03$
\end{tabular} & - & $\begin{array}{l}65.4 \pm 5.8 \\
-0.55 \pm 0.01\end{array}$ & $0.50 \pm 0.04$ \\
\hline
\end{tabular}


For probe wavelength $580 \mathrm{~nm}$ probing the CdSe bleach feature also in the metal tipped samples a second formation time appears, which is much shorter than for the bare rod and in the order of magnitude of the electron injection time from the CdS excitonic state to the metal nanoparticle. This reflects the situation, that the exciton relaxation to the seed occurs in concurrence to electron injection and as localization is slower than the injection, injection limits the timescale on which exciton localization dynamics is observable.

Metal-tipped samples - Au

The model applied to fit the data of the Au-tipped sample is similar to the one described above.

$$
\Delta A(t)=A_{e-p h} e^{\frac{-t}{\tau_{e-p h}}}+c *\left(\sum_{i} A_{f i} e^{\frac{-t}{\tau_{f i}}}+\sum_{i} A_{i} e^{\frac{-t}{\tau_{i}}}\right)+\text { const }
$$

$\mathrm{A}_{\mathrm{e}-\mathrm{ph}}, \tau_{\mathrm{e}-\mathrm{ph}}$ amplitude and time constant of signal formation processes

Table S4: fit parameters for electron-phonon relaxation in the Au tipped sample

\begin{tabular}{|l|l|l|}
\hline Au & $\begin{array}{l}\boldsymbol{\tau}_{\text {e-ph }} / \mathbf{p s} \\
\mathbf{A}_{\text {e-ph }}\end{array}$ & $\mathbf{C}$ \\
\hline $\begin{array}{l}458 \mathrm{~nm} \\
\mathrm{CdS} \\
\text { (rod/shell) }\end{array}$ & $\begin{array}{l}2.5 \pm 0.6 \\
-0.09 \pm 0.01\end{array}$ & $0.75 \pm 0.01$ \\
\hline $\begin{array}{l}505 \mathrm{~nm} \\
\mathrm{Au} \mathrm{SPR}\end{array}$ & $\begin{array}{l}2.2 \pm 0.1 \\
-1.66 \pm 0.02\end{array}$ & $0.93 \pm 0.03$ \\
\hline $\begin{array}{l}580 \mathrm{~nm} \\
\mathrm{CdSe}\end{array}$ & $\begin{array}{l}2.6 \pm 0.3 \\
0.6 \pm 0.03\end{array}$ & $1.16 \pm 0.04$ \\
\hline
\end{tabular}

The dynamics in the Au-tipped sample is a simple superposition of Au nanoparticle intrinsic dissipation processes and semiconductor intrinsic recombination processes. The maximum of the contributions of the $2.2 \mathrm{ps}$ component at the maximum of the SPR bleach feature indicated this process to be related to relaxation within the Au nanoparticle. 


\section{Literature}

1. Siebert, R.; Akimov, D.; Schmitt, M.; Winter, A.; Schubert, U. S.; Dietzek, B.; Popp, J. Spectroscopic Investigation of the Ultrafast Photoinduced Dynamics in pi-Conjugated Terpyridines. ChemPhysChem 2009, 10, 910-919.

2. Barthelmes, K.; Kübel, J.; Winter, A.; Wächtler, M.; Friebe, C.; Dietzek, B.; Schubert, U. S. New Ruthenium Bis(terpyridine) Methanofullerene and Pyrrolidinofullerene Complexes: Synthesis and Electrochemical and Photophysical Properties. Inorg. Chem. 2015, 54, 3159-3171.

3. Wu, K. F.; Zhu, H. M.; Liu, Z.; Rodriguez-Cordoba, W.; Lian, T. Q. Ultrafast Charge Separation and Long-Lived Charge Separated State in Photocatalytic CdS-Pt Nanorod Heterostructures. J. Am. Chem. Soc. 2012, 134, 10337-10340.

4. Wu, K. F.; Rodriguez-Cordoba, W. E.; Liu, Z.; Zhu, H. M.; Lian, T. Q. Beyond Band Alignment: Hole Localization Driven Formation of Three Spatially Separated Long-Lived Exciton States in CdSe/CdS Nanorods. ACS Nano 2013, 7, 7173-7185. 